\title{
Integrated Sensing for IPMC Actuators Using Strain Gages for Underwater Applications
}

\author{
Kam K. Leang, Member, IEEE, Yingfeng Shan, Sisi Song, and Kwang J. Kim
}

\begin{abstract}
Ionic polymer-metal composite (IPMC) actuators have many advantages, for instance, they: 1) can be driven with low voltages $(<5 \mathrm{~V}) ; 2)$ are soft, flexible, and easily shaped; and 3) can operate in an aqueous environment (such as water). Important applications for IPMCs include active catheter devices for minimally invasive surgery, artificial muscles, and sensors and actuators for biorobotics. Due to inherent nonlinear behavior, dynamic effects, and external disturbances, sensing and feedback control are required for precision operation. A new method to sense the displacement of an IPMC actuator using resistive strain gages is proposed. The sensing scheme is low cost, practical, effective, and importantly, compact compared to existing methods such as lasers and charge-coupled device (CCD) cameras. The strain-to-displacement relationship is developed and experimental results are presented to demonstrate the effectiveness of the sensing scheme. Furthermore, the sensor signal is used as feedback information in a repetitive controller to improve the tracking of periodic motion. The stability condition for the controller is presented, and the sensing scheme and feedback control approach are applied to a fabricated perfluorinated ion-exchange-membrane-based IPMC actuator with lithium as its counterion. Experimental results show that the tracking error can be reduced by approximately $50 \%$ compared to PID control for tracking of periodic signals, including sinusoidal and triangular wave forms.
\end{abstract}

Index Terms-Ionic polymer-metal composite (IPMC) actuators, repetitive control, strain gage sensors.

\section{INTRODUCTION}

I ONIC polymer-metal composites (IPMCs) are innovative materials that can be exploited for emerging robotic, intelligent mechatronic, bioinpsired, and biomedical systems [1]-[5]. Due to their low driving voltage $(<5 \mathrm{~V})$, large strain, soft and flexible structure, and the ability to operate in an aqueous environment (such as water), IPMCs are suitable for many unique applications that include innovative propulsion systems in underwater autonomous systems [6]-[9]. Particularly, strips of IPMCs have been used to construct the legs (tentacles) of a jellyfish-

Manuscript received August 6, 2010; revised November 4, 2010 and November 15, 2010; accepted November 21, 2010. Date of publication February 14, 2011; date of current version January 20, 2012. Recommended by Technical Editor G. Alici. This work was supported in part by the Office of Naval Research under Grant N000140910218.

K. K. Leang, Y. Shan, and S. Song are with the Controls (EASY) Laboratory and the Mechanical Engineering Department, University of Nevada, Reno, NV 89557-0312 USA (e-mail: kam@unr.edu).

K. J. Kim is with the Active Materials and Processing Laboratory (AMPL) and the Mechanical Engineering Department, University of Nevada, Reno, NV 89557-0312 USA (e-mail: kwangkim@unr.edu).

Color versions of one or more of the figures in this paper are available online at http://ieeexplore.ieee.org.

Digital Object Identifier 10.1109/TMECH.2011.2105885 like robot [10]. The walking speed of the jellyfish robot was controlled through the frequency of the input voltage applied to the IPMC-based legs. Likewise, the caudal fin to propel a robotic fish was created from an IPMC with an achievable peak swimming speed reported at $22 \mathrm{~mm} / \mathrm{s}$ [11]. However, IPMC actuators can play a critical role in the development of highlymaneuverable biorobotic vehicles, e.g., the system described in [12].

Having the capability to measure the performance of the actuator is critical for modeling [13], [14] and active control of IPMC actuators to compensate for nonlinearities, dynamic effects, and external disturbances [15]-[17]. The sensing methods for IPMCs can be grouped into two broad categories: 1) integrated or 2) nonintegrated sensors. In the first category, the sensor is either part of the composite material (self-sensing), permanently attached, or embedded within the composite material. For example, the mechanoelectrical transduction ability of IPMC materials can be exploited to create IPMC-based sensors, such as for prostheses [2]. Because IPMCs can function both as a sensor and actuator [18], an IPMC with patterned electrodes for self-sensing has been proposed [19]-[21]. This method involves creating two isolated regions of the composite material, one for actuation and one for sensing deformation. The selfsensing approach is compact; however, the major challenges are ensuring that the actuating and sensing electrodes are properly isolated to overcome feedthrough or cross-coupling issues [20], [21] and accounting for sensor nonlinearity [18]. Another example is bonding polyvinylidene fluoride (PVDF) thin films to the surface of an IPMC actuator [22]. In this case, thin-film sensors are preferred to minimize the effect of the attached sensor on the natural behavior of the IPMC actuator during normal operation. However, feedthrough between the applied voltages to the IPMC electrodes and the attached sensor via the insulating layer must be carefully considered [22].

Nonintegrated sensors include lasers [23], charge-coupled device (CCD) cameras [24], or force sensors [25] that may or may not come into physical contact with the actuator, to measure the IPMC's response for feedback control. Laser displacement sensors are by far the most common because they are ubiquitous and easy to use. The sensing range for lasers typically falls between $1 \mathrm{~mm}$ and several hundred millimeters, with resolution down to $1 \mu \mathrm{m}$, and the bandwidth is up to a few kilohertz. Most laser displacement sensors use the principle of optical triangulation. A visible modulated point of light is projected onto the target surface, such as the platinum electrode of an IPMC actuator. An optical receiver element, for example, a high-sensitivity resolution element (CCD array), positioned at a certain angle to the optical axis of the laser then images the diffused light spot. A 


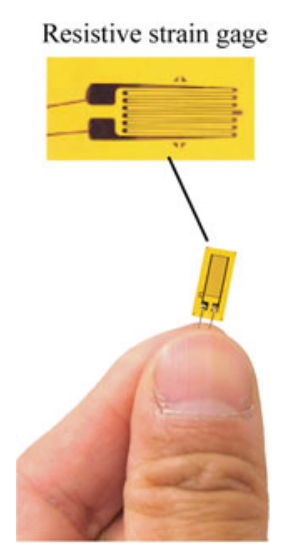

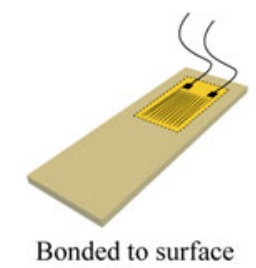

Bonded to surface

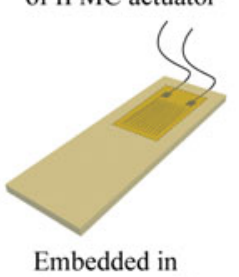

polymer material of IPMC actuator
Fig. 1. Resistive strain gage for IPMC actuators.

controller calculates the distance based on the measurement of the CCD array. Laser displacement sensors are relatively inexpensive and the light beam can penetrate water as well as many types of transparent layers, such as acrylic plastic. Practically speaking, however, the disadvantage is the sensor head and controller electronics are bulky, thus precluding their use in small and lightweight autonomous systems. Additionally, the output can vary with surface reflectivity. Charge-coupled device cameras and computer software can be used to estimate the IPMC's deformation [24]. Like lasers, they too are bulky and not practical for microautonomous systems. A more compact approach is the capacitance-based method that locates the functioning IPMC between two parallel plates [26]. In general, the majority of nonintegrated sensors are too bulky for sensing and control in small IPMC-based autonomous systems. The need for a more compact and low-cost solution motivates this paper and in the following, strain gages are proposed as a viable sensing scheme for IPMCs.

The contribution of this paper is the integration of a resistive strain gage with an IPMC actuator to create a compact actuator/sensor system. Therefore, traditionally used sensors such as lasers, which are sensitive to surface reflectivity and bulky, are not required for feedback control. As shown in Fig. 1, small resistive strain gages can either be glued to the surface of an IPMC actuator or embedded into the polymer material through solution casting or other fabrication methods. Aside from being compact, these sensors are also flexible, lightweight, and low power, and have high resolution and sufficient bandwidth. Multiple strain gages can be used to sense deformation in multiple directions, such as in IPMCs capability of bending and twisting motion [27]. Likewise, strain gages can be potentially integrated with emerging IPMC manufacturing techniques [28]. The application of the strain gage is further demonstrated by developing a discrete-time repetitive controller (RC) that acts on the measured signal from the strain gage for tracking of periodic reference trajectories. It is pointed out that any control approach that requires real-time information of the IPMC's deformation can benefit from the proposed sensing scheme, for example, robust and adaptive feedback control [15], [16]. However, the $\mathrm{RC}$ approach is specifically chosen because it is well-suited for applications where the actuator's motion is required to be periodic in time. A good example of this is the oscillatory motion of an IPMC-based caudal fin for underwater propulsion [29]. In general, most applications that require an IPMC actuator to move in a periodic fashion, including active endoscopes [3], can benefit from the RC approach. The fabrication of the compact actuator/sensor system and a model for estimating the actuator's displacement given a strain value are discussed. In addition, the limitation of the strain gage sensing scheme is also highlighted.

\section{IPMC MANUfACtURING PROCESS}

IPMC actuators used for the experiments are customfabricated by using standard techniques [6], [30]. Specifically, the perfluorinated ion exchange membrane is created by using a hot press and molding process, followed by applying a layer of platinum on the outer surface of the membrane using an electroless plating process, then infusing lithium as a counterion. First, a pretreatment is performed on the ion exchange membrane prior to electroding to eliminate organic and metallic impurities. Basically, the membrane is heated to $80^{\circ} \mathrm{C}$ in $3 \%$ hydrogen peroxide $\left(\mathrm{H}_{2} \mathrm{O}_{2}\right)$ and $15 \%$ nitric acid $\left(\mathrm{HNO}_{3}\right)$. Next, the membrane is immersed for $3 \mathrm{~h}$ in the platinum complex solution prepared with tetraamineplatinum (II) chloridemonohydrate $\left(\left[\mathrm{Pt}\left(\mathrm{NH}_{3}\right)_{4}\right] \mathrm{Cl}_{2} \cdot \mathrm{H}_{2} \mathrm{O}\right.$, Aldrich). Subsequently, the membrane is rinsed with deionized water. For the initial metalization of the membrane surface with platinum particles, the membrane is reduced in a sodium borohydride $\left(\mathrm{NaBH}_{4}\right.$, Aldrich) solution for $3 \mathrm{~h}$. The membrane is additionally run through the platinum electroding process to increase the surface conductivity. Finally, the $\mathrm{H}^{+}$cation within the membrane is exchanged to $\mathrm{Li}^{+}$by using $1 \mathrm{M}$ lithium chloride ( $\mathrm{LiCl}$, Aldrich) solution, soaking the IPMC for $24 \mathrm{~h}$.

\section{A. Control Hardware Design and Dynamics Model}

A custom voltage/current amplifier is developed to control the bending motion of the IPMC actuator. Fig. 2(a) and (b) shows the circuit block diagram and fabricated circuit board ${ }^{1}$ for the amplifier, respectively. The amplifier is a class-B, emitter-follower design with feedback to minimize crossover distortion [31]. In voltage mode, the voltage difference across the electrodes of the actuator is used as feedback information, as shown in Fig. 2(a). In current mode, resistor $R_{s}(1 \Omega, 5 \mathrm{~W})$ functions as a current sensor. The measured unloaded bandwidth $(-3.01 \mathrm{~dB})$ in voltage mode exceeds $100 \mathrm{kHz}$. Likewise, the measured crossover distortion at different input frequencies using a $1 \mathrm{~V}$ triangle input signal for a $4 \Omega$ resistive load is shown in Fig. 2(c). At frequencies less than $1000 \mathrm{~Hz}$, the maximum crossover distortion is less than $2 \%$.

The custom-designed voltage amplifier is used to drive the fabricated IPMC actuator (dimensions: effective length $L=$ $45 \mathrm{~mm}, w=15 \mathrm{~mm}$, and $t=0.5 \mathrm{~mm}$ ), and the open-loop

\footnotetext{
${ }^{1}$ Circuit diagram is available by contacting the corresponding author (K. K. Leang, e-mail: kam@unr.edu).
} 
(a)

(b)

(c)
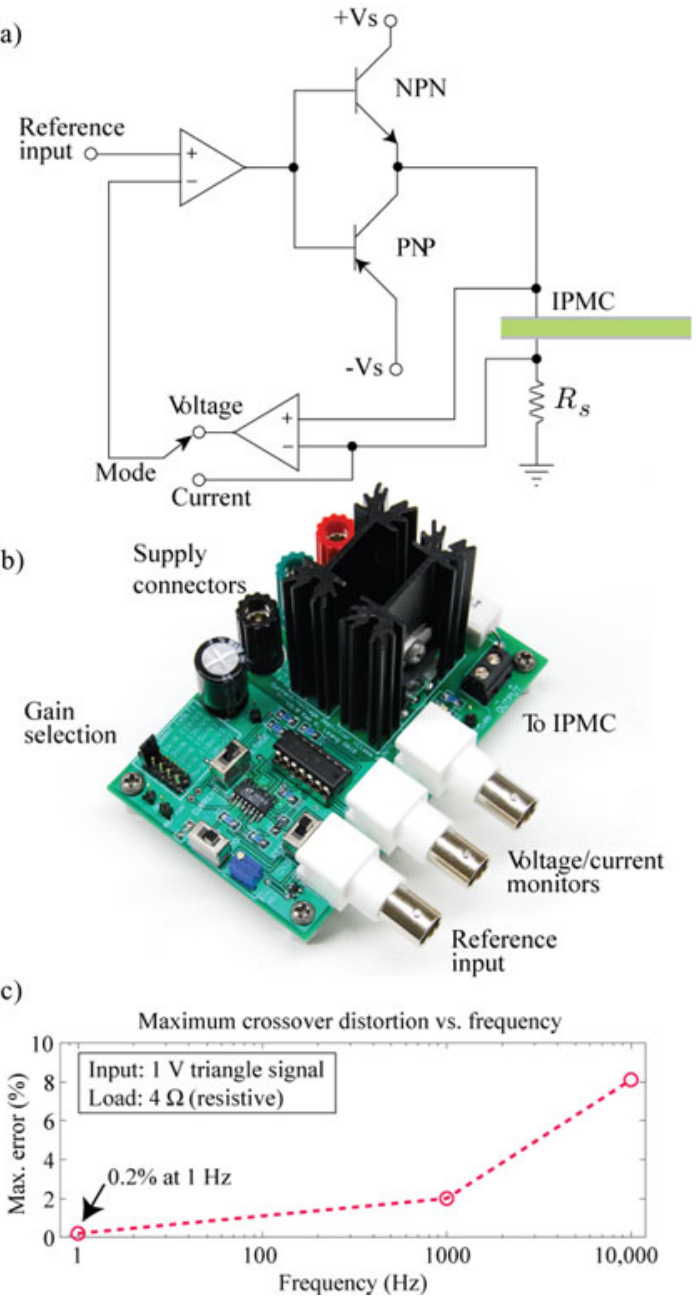

Fig. 2. Custom design voltage/current amplifier for IPMC actuator. (a) Circuit diagram. (b) Fabricated circuit board. (c) Measured crossover distortion versus frequency.
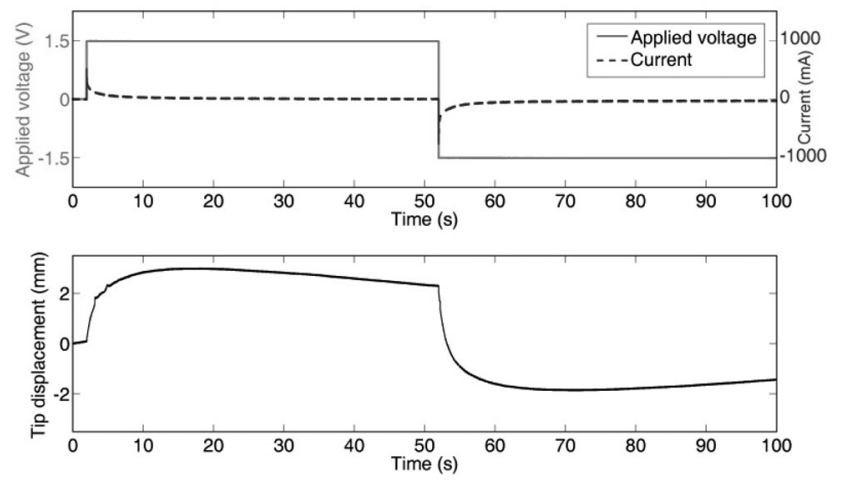

Fig. 3 Voltage, current, and open-loop tip response of $\mathrm{Li}^{+}$-based IPMC actuator (measured with laser sensor).

response of the actuator to a square wave input is shown in Fig. 3, measured with a laser displacement sensor (Micro-Epsilon, optoNCDT 1402). The IPMC initially responds by bending to a maximum value, then slowly back-relaxes over a period of $50 \mathrm{~s}$ [6]. It is noted that in the experiments, two IPMCs with thickness of 0.5 and $1.0 \mathrm{~mm}$ are used. (a)

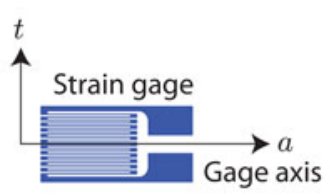

(b)

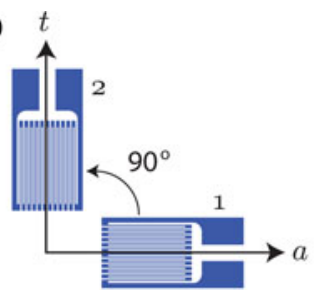

(c)

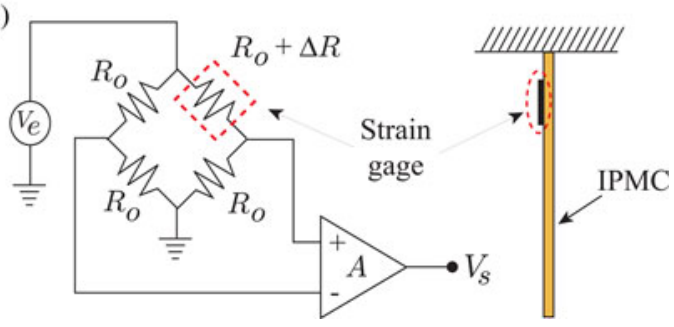

Fig. 4. Strain gage for IPMC. (a) Strain gage. (b) Two gages mounted orthogonal to each other. (c) Quarter-bridge strain gage circuit.

\section{Strain Gage for DisPlacement Measurement}

\section{A. Governing Equations}

For position control of active material actuators including piezoelectric actuators, resistive strain gages are commonly used [32], [33]. One of the advantages of strain gages is that they are compact, thin, and flexible and can easily be bonded to an actuator's surface. Resistive strain gages are made of either a small-diameter wire or thin layer of conducting foil encapsulated in an insulating material. When used with an IPMC actuator, the packaging eliminates cross-coupling effects between the applied voltage to the IPMC electrodes and the attached strain gage.

A standard strain gage with nominal resistance $R_{o}$ is shown in Fig. 4(a), where it is aligned such that the gage axis is parallel to the axial direction indicated by $a$. When the (wire or foil) gage is elongated, the resistance changes with respect to the strain field. Therefore, the unit change in resistance is

$$
\frac{\Delta R}{R_{o}}=F_{a} \epsilon_{a}
$$

when the transverse strain $\epsilon_{t}$ is zero. Likewise,

$$
\frac{\Delta R}{R_{o}}=F_{t} \epsilon_{t}
$$

when the axial strain $\epsilon_{a}$ is zero. The constants $F_{a}$ and $F_{t}$ are the axial and transverse strain sensitivities, respectively. On the other hand, when a gage is calibrated in a biaxial strain field in which the gage is mounted to a material of known Poisson ratio (e.g., $\nu_{o}=0.285$ ) in accordance with ASTM Standard E25186(12), the unit change in resistance is

$$
\frac{\Delta R}{R_{o}}=G_{f} \epsilon_{a}
$$

where $G_{f}$ is the gage factor, a value that is typically provided by the strain gage manufacturer [34]. In general, when the gage is subjected to strains in both the axial and transverse directions, the total change in resistance is obtained by the sum of (1) and 
(2); hence,

$$
\frac{\Delta R}{R_{o}}=F_{a}\left(\epsilon_{a}+K \epsilon_{t}\right)
$$

where $K=F_{t} / F_{a}$ is known as the transverse sensitivity factor, a value often provided by the manufacturer or it can be determined experimentally [35], [36]. The transverse sensitivity factor indicates the effect of the transverse strain on the total indicated strain from the gage. This value can be positive or negative and depends on many factors including the material of the wire or foil gage. It is emphasized that if the value of $K$ for a given strain gage is greater than $3 \%$ and the traverse strain exceeds the axial strain, then an error greater than $4 \%$ will result [34]. The gage factor and the axial and transverse strain sensitivities are related by setting $\epsilon_{t}=-\nu_{o} \epsilon_{a}$, and then, equating (3) and (4); hence,

$$
F_{a}=\frac{G_{f}}{1-\nu_{o} K} \quad \text { and } \quad F_{t}=\frac{K G_{f}}{1-\nu_{o} K} .
$$

If a single gage is used to measure strain under biaxial conditions differing from those of calibration, an error will result [34]. Particularly, in IPMCs, it is believed that deformation is caused by swelling-induced strain in the boundary layers near the electrode region [37], [38]. The swelling effect can be biaxial in nature and occurs due to migration of water molecules in the boundary layer. Therefore, if a strain gage is mounted to the surface of an IPMC actuator, the biaxial nature of the induced strain and the transverse sensitivity factor, if significant, must be taken into account. To do so, two identical strain gages, labeled as 1 and 2, are mounted orthogonal to each other, as shown in Fig. 4(b). Specifically, gage 1 is aligned in the direction of the desired strain and gage 2 is aligned normal to the desired strain direction. When the gages are subjected to an unknown biaxial stress field, application of (3) to (5) results in the following relationships [34]:

$$
\begin{aligned}
\epsilon_{1} & =\frac{\left(1-\nu_{o} K\right)\left(\bar{\epsilon}_{1}-K \bar{\epsilon}_{2}\right)}{1-K^{2}} \\
\epsilon_{2} & =\frac{\left(1-\nu_{o} K\right)\left(\bar{\epsilon}_{2}-K \bar{\epsilon}_{1}\right)}{1-K^{2}}
\end{aligned}
$$

where $\bar{\epsilon}_{1}$ and $\bar{\epsilon}_{2}$ are the indicated axial strains for gages 1 and 2, respectively. It is noted that if the transverse sensitivity factor is ignored $(K=0)$, then $\epsilon_{1}=\bar{\epsilon}_{1}$ and $\epsilon_{2}=\bar{\epsilon}_{2}$. The indicated axial strains in terms of the unit change in resistances are

$$
\bar{\epsilon}_{i}=\frac{1}{G_{f}}\left(\frac{\Delta R}{R_{o}}\right)_{i}
$$

for $i=1,2$. Substituting (8) into (6) and (7), the unit change in resistances for gages 1 and 2 as a function of the actual axial strains are

$$
\begin{aligned}
& \left(\frac{\Delta R}{R_{o}}\right)_{1}=G_{f}\left(\frac{\epsilon_{1}+K \epsilon_{2}}{1-\nu_{o} K}\right) \\
& \left(\frac{\Delta R}{R_{o}}\right)_{2}=G_{f}\left(\frac{\epsilon_{2}+K \epsilon_{1}}{1-\nu_{o} K}\right) .
\end{aligned}
$$

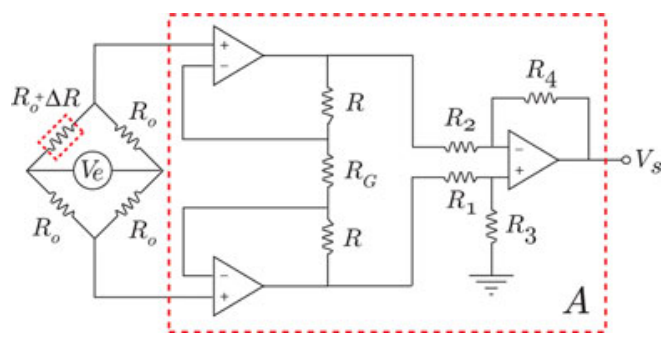

Fig. 5. Strain gage amplifier.

\section{B. Circuitry}

Due to the minute changes in resistance corresponding to strain, the strain gage is often connected to an electric circuit such as a Wheatstone bridge. For instance, the quarter-bridge circuit shown in Fig. 4(c) produces a change in the output voltage $V_{1 / 4}$ as a function of the unit change in resistance $\Delta R / R_{o}$ of the strain gage

$$
V_{\frac{1}{4}}=\left(\frac{\Delta R / R_{o}}{2}\right) \times\left(\frac{1}{2+\Delta R / R_{o}}\right) V_{e}
$$

where $V_{e}$ is the gage excitation voltage. The gage excitation voltage is kept relatively low $(\leq 5 \mathrm{~V})$ to avoid self-heating that can degrade the performance of the gage [39]. The thermal conductivity of the surrounding environment should also be taken into consideration, especially when gages are used with IPMCs as they are commonly operated in an aqueous environment, such as water. However, temperature compensating can be accomplished by using two gages [34]. By neglecting the bridge nonlinearity, the change in the output voltage as a function of the unit change in resistance is

$$
V_{\frac{1}{4}}=\frac{1}{4}\left(\frac{\Delta R}{R_{o}}\right) V_{e} .
$$

Likewise, for a half-bridge circuit, $V_{1 / 2}=2 V_{1 / 4}$.

Since the achievable strain for an IPMC actuator is typically less than $1 \%$, the change in resistance of the gage is relatively small, and thus, the bridge voltage is amplified by the differential amplifier, as shown in Fig. 5. By selecting $R_{1}=R_{2}$ and $R_{3}=$ $R_{4}$, the output voltage is

$$
V_{s}=B A\left(\frac{\Delta R}{R_{o}}\right) V_{e}
$$

where $A=\left[R_{3} / R_{1}\left(2 R /\left(R_{G}\right)+1\right)\right]$ is the amplifier gain, $B=$ $1 / 4$ for a quarter-bridge circuit, and $B=1 / 2$ for a half-bridge circuit. It is pointed out that strain gages can detect relatively high-frequency strains, and the bandwidth of the sensor is often limited by the bandwidth of the signal conditioning circuitry (amplifier circuit). In the experiments to follow, the bandwidth of the strain amplifier is $10 \mathrm{kHz}$, which is significantly higher than the actuator's positioning bandwidth.

When two identical gages are used and arranged, as shown in Fig. 4(b), the true axial strain in each gage, $\epsilon_{1}$ and $\epsilon_{2}$, as a function of the gage characteristics and circuit parameters can be obtained by substituting (9) and (10) into (13). Specifically, 
the axial strains for gage 1 and gage 2 are

$$
\begin{aligned}
& \epsilon_{1}=\left(\frac{B^{-1}}{G_{f} A V_{e}}\right) \times\left(\frac{1-\nu_{o} K}{1-K^{2}}\right) \times\left(V_{s, 1}-K V_{s, 2}\right) \\
& \epsilon_{2}=\left(\frac{B^{-1}}{G_{f} A V_{e}}\right) \times\left(\frac{1-\nu_{o} K}{1-K^{2}}\right) \times\left(V_{s, 2}-K V_{s, 1}\right)
\end{aligned}
$$

where $V_{s, 1}$ and $V_{s, 2}$ are the indicated axial strains, i.e., measured strain signals from gage 1 and gage 2 , respectively.

\section{Mounting Considerations}

A widely used general-purpose adhesive for attaching strain gages is cyanoacrylate-based fast-acting adhesive such as those sold under trade names like Super Glue and Krazy Glue. Cyanoacrylate-based adhesives are simple to use, can withstand strains up to $15 \%$, require no mixing, and can operate over a temperature range of -32 to $65^{\circ} \mathrm{C}$. Unfortunately, the adhesive is sensitive to moisture and a protective coating is required for wet environments. Additionally, it is not recommended for use over long periods of time due to embrittlement with age. Epoxies, such as the type where polymerization occurs at room temperature or those that require external heat for polymerization, are often preferred for standard applications because of their excellent moisture and chemical resistance, minimal shrinkage, and good bonding strength with a wide variety of materials. Epoxy adhesives can operate over a wide range of temperatures, from cryogenic to levels beyond $300^{\circ} \mathrm{C}$. In this paper, rubber-based water insoluble adhesive with toluene solvent (such as Surebonder 9001 or Loctite Stik'n Seal Original) is used for mounting the strain gage to the surface of the IPMC actuator. This type of adhesive is chosen because of its superior water resistance and flexibility upon cure to avoid delamination problems. A simple experiment is done to determine the shear stress at failure. A small sample of Kapton film (approximately $500 \mathrm{~mm}^{2}$ ) is used to simulate the strain gage, then it is attached to an IPMC sample with platinum electrodes. Upon curing, the glued samples are allowed to soak in water for $12 \mathrm{~h}$, and then, are pulled apart. The shear stress at failure fell between 179 and $246 \mathrm{kPa}$ for the two adhesives mentioned earlier. In contrast, other adhesives including marine epoxy failed at as low as $33 \mathrm{kPa}$.

The procedures for mounting the gage are as follows. First, the IPMC actuator is sufficiently hydrated in deionized water (approximately $12 \mathrm{~h}$ ), then the surface of the actuator is quickly dried with a clean and dry cloth. Immediately following, the surfaces of the strain gage and the IPMC electrode are cleaned, for example, using isopropyl alcohol. Next, both surfaces are coated with a thin layer of the adhesive. The gage is applied to the surface of the IPMC and held in place under pressure using a small clamp for $12 \mathrm{~h}$. Afterward, a resistance meter is used to check for proper electrical isolation among the gage, leads, and the IPMC's electrode. The recommended resistance is $10 \mathrm{k} \Omega$ minimum [34]. Prior to use, wires are carefully soldered and all exposed electrical surfaces are waterproofed by coating them with a thin layer of adhesive to minimize feedthrough between the applied input voltage and strain signal. The integrated IPMC actuator/sensor device is then soaked in water for at least $12 \mathrm{~h}$ before use. It is noted that attaching the gage to a hydrated IPMC

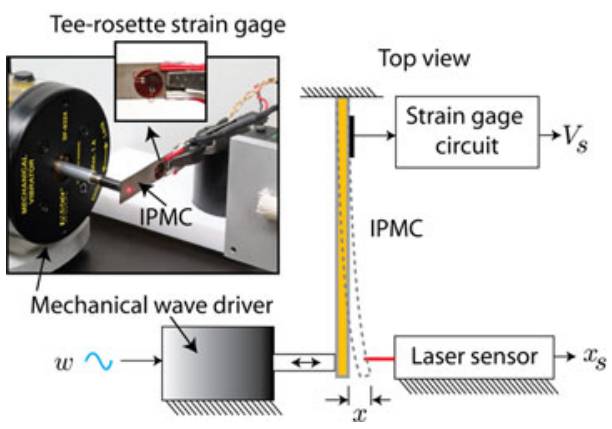

Fig. 6. Experimental setup using mechanical wave driver to displace IPMC cantilever to compare the displacement measured using a laser sensor and the strain gages' response. A $0^{\circ} / 90^{\circ}$ tee-rosette strain gage is used to measure the axial and transverse strains.

minimizes the effects of excessive shrinkage of the material as the adhesive cures. Additionally, gages are mounted to the top and bottom surfaces of the IPMC to form a half-bridge configuration that further helps to compensate the effects of induced curvature due to material shrinkage. The effects of shrinkage when the gage is mounted can induce residual strains, and this effect presents a major challenge. Future study will focus on embedding the strain gage into the polymer material during the fabrication process to address this problem.

\section{Measured Performance}

Experiments are performed to study the feasibility of the strain gage for sensing displacement of an IPMC actuator mounted in the cantilever configuration. Fig. 6 shows a photograph of a small $0^{\circ} / 90^{\circ}$ tee-rosette strain gage package (Omega KFG-3-350-D16-11L1M2S, $R_{o}=350 \Omega$, gage length of $3 \mathrm{~mm}$, $G_{f}=2.10 \pm 1.0 \%$, and $K \approx 0.02$ ) bonded to the surface of a $45 \mathrm{~mm} \times 15 \mathrm{~mm} \times 1 \mathrm{~mm}$ IPMC actuator near its clamped end. The tee-rosette gage consists of two strain gages mounted orthogonal to each other to provide axial and transverse strain measurement. For the first experiment, a mechanical wave driver (Pasco, model SF-9324) is used to displace the free end of the IPMC actuator in air, where $w$ represents the input signal to the wave driver. The movement of the free end of the cantilever, $x$, is measured by a laser sensor (Micro-Epsilon, model optoNCDT 1402) for comparison. The mechanical wave driver displaces the IPMC cantilever over a range of $\pm 0.5 \mathrm{~mm}$ in a sinusoidal manner at $0.1,1,5$, and $10 \mathrm{~Hz}$. The signals from the laser sensor $x_{s}$ and the strain gage $V_{s}$ are compared on a normalized scale (equivalent to tip displacement of $\pm 0.5 \mathrm{~mm}$ ), as shown in Fig. 7. The results show good correspondence between the strain signal and tip displacement measured by the laser. However, at relatively low frequencies (e.g., $0.1 \mathrm{~Hz}$ ), the strain signal exhibits drifting effect as well as its phase leads the displacement signal. The low-frequency phase lead of the strain signal is believed to be attributed to viscoelastic effects of the polymer membrane.

In the second experiment, a square wave input voltage (amplitude $1 \mathrm{~V}$ ) is applied to drive the IPMC actuator in both air and water. Fig. 8 compares the resulting bending motion measured by the laser sensor (dash line) and strain gage (solid line). Both the strain gage's and laser sensor's response are virtually 

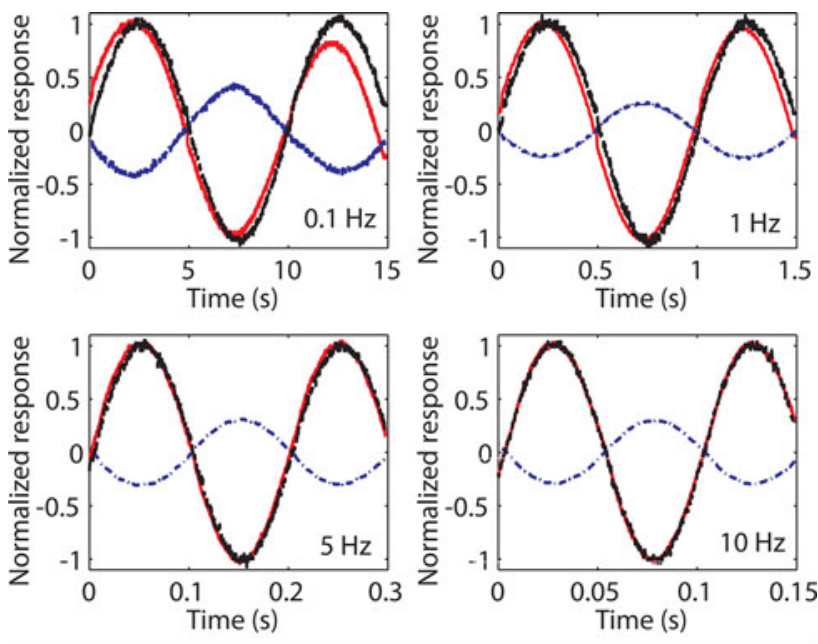

_ Axial strain _.-. Transverse strain _.-- Displacement

Fig. 7. Response of mechanically displaced IPMC $(45 \mathrm{~mm} \times 15 \mathrm{~mm} \times 1.0 \mathrm{~mm}$ sample) in air, comparing the displacement measured by the laser sensor to the response of the strain gages (axial and transverse).

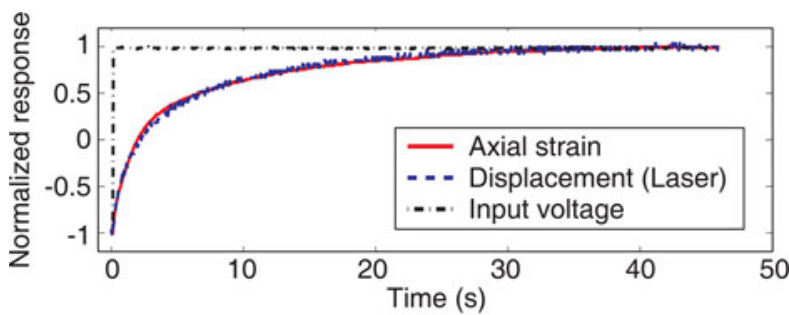

(a)

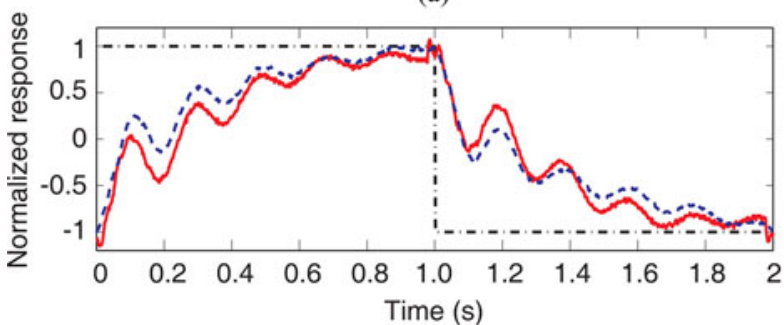

(b)

Fig. 8. Response of electrically-activated IPMC: (a) $45 \mathrm{~mm} \times 15 \mathrm{~mm} \times$ $1.0 \mathrm{~mm}$ sample operating in air and (b) $45 \mathrm{~mm} \times 15 \mathrm{~mm} \times 0.5 \mathrm{~mm}$ sample operating in deionized water comparing the displacement measured with a laser sensor to the response of the axial strain gage $(0.5 \mathrm{~Hz}$ square wave input signal).

indistinguishable for measuring the IPMC's low-frequency bending response, as indicated in Fig. 8(a), operating in air. A good match is also observed at a higher frequency $0.5 \mathrm{~Hz}$ input signal, operating in water, as shown in Fig. 8(b). In this case, both sensors recorded the dominant oscillating behavior of the actuator. The oscillatory behavior is caused by the actuator's mechanical resonance at approximately $10 \mathrm{~Hz}$ (see Fig. 9). However, a notable difference exists due to the effects of higher order modes. The difference in the sensors' response is also noted in the measured frequency response above $10 \mathrm{~Hz}$, as shown in Fig. 9.
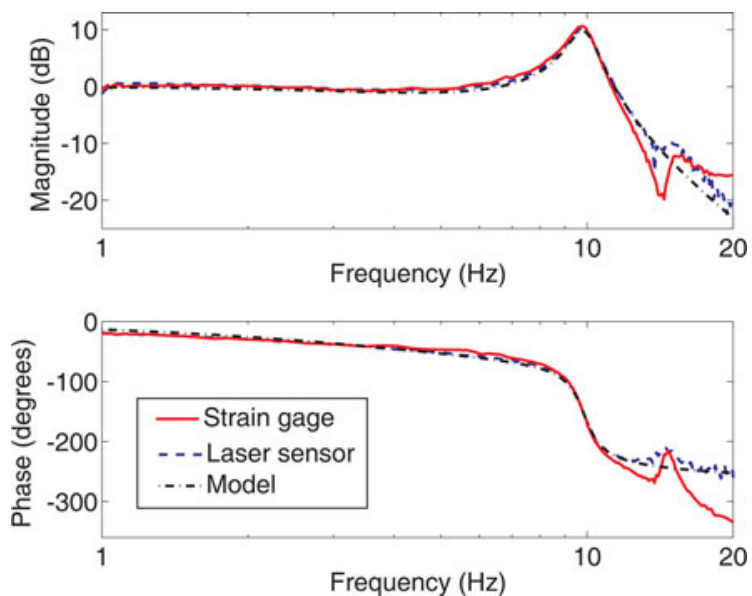

Fig. 9. Frequency response of electrically-activated IPMC ( $45 \mathrm{~mm} \times 15 \mathrm{~mm} \times$ $0.5 \mathrm{~mm}$ sample) in water: measured using the strain gage (solid line) and laser sensor (dashed line) compared to the linear dynamics model (dash-dot). (a)

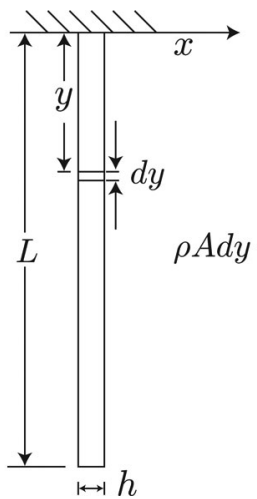

(b)

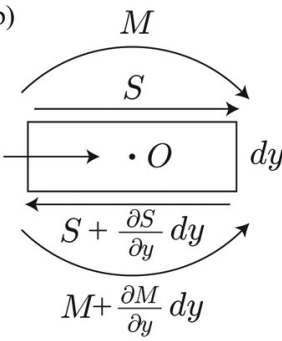

Fig. 10. (a) Cantilever beam. (b) Free body diagram of beam element.

\section{E. Strain-to-Displacement Relationship}

For a typical IPMC strip actuator mounted in the cantilever configuration, the control variable is often the tip displacement. A strain-to-displacement relationship is developed to predict the bending displacement of the IPMC actuator from the measured strain. The analysis assumes that the IPMC's bending response follows that of a isotropic cantilever beam, as shown in Fig. 10(a) [40], [41]. Let $x(y, t)$ denote the displacement from the neutral axis of an element at the distance $y$ from the fixed end [see Fig. 10(b)]. Given a strain measurement $\epsilon\left(y_{1}, t\right)$ at location $y_{1}$ and time $t$, the objective is to determine the transverse displacement $x\left(y_{d}, t\right)$ at $y_{d}$ and time $t$ for feedback control.

For the cantilever beam shown in Fig. 10(a), it is assumed that the following general equation of motion holds:

$$
\rho A \frac{\partial^{2} x(y, t)}{\partial t^{2}}+c(y) \frac{\partial x(y, t)}{\partial t}+E I \frac{\partial^{4} x(y, t)}{\partial y^{4}}=F(y, t)
$$

where $\rho$ is the density, $A$ is the cross-sectional area, $E$ is the Young's modulus, $I$ is the moment of inertia, and $c(y)$ and $F(y, t)$ are the damping coefficient and applied load per unit length, respectively. The solution, the transverse displacement, to the equation of motion (16) is obtained by separating $x(y, t)$ into two independent components: spatial and temporal. 
Therefore, the total solution is the superposition of the motion of each natural mode $\chi_{n}(y)$ of the beam

$$
x(y, t)=\sum_{n=1}^{\infty} \chi_{n}(y) \phi_{n}(t)
$$

where $n$ indexes the modes and $\phi_{n}(t)$ composes of the harmonic oscillations of the natural modes. The solutions to $\chi_{n}(y)$ and $\phi_{n}(t)$ depend on the boundary and initial conditions for the beam, respectively, [42].

To relate the strain to the transverse displacement of the beam, consider that the tensile stress $\sigma(y, t)$ at a point of the beam is equal to $-M(y, t) c / I$, where $c$ is the distance from the point to the neutral axis. Hence, from Hooke's Law for pure bending

$$
E \epsilon(y, t)=\sigma(y, t)=-\frac{M(y, t) c}{I}=-\left(E I \frac{\partial^{2} x(y)}{\partial y^{2}}\right) \frac{c}{I} .
$$

Therefore, the strain at the surface of the beam, a distance of $c=h / 2$ from the neutral axis, is related to the transverse displacement by

$$
\epsilon(y, t)=-\frac{h}{2} \times \frac{\partial^{2} x(y, t)}{\partial y^{2}}=-\frac{h}{2} \sum_{n=1}^{\infty} \frac{\partial^{2} \chi_{n}(y)}{\partial y^{2}} \phi_{n}(t) .
$$

If it is assumed that the motion of the cantilevered IPMC can be approximated by a finite number of modes, $\partial^{2} \chi_{n}(y) / \partial y^{2}$ can be calculated, allowing for the solution of $\phi_{n}(t)$ given the strain measurement $\epsilon(y, t)$. Finally, the transverse displacement $x(y, t)$ is found by (17).

To illustrate the application of the aforementioned method, consider the measured frequency response shown by the dash line in Fig. 9. The response is measured near the free end of the cantilever, $y \approx y_{d}$, using the laser displacement sensor. The result indicates that the displacement is heavily dominated by the first mode up to a frequency of $20 \mathrm{~Hz}$; therefore

$$
\begin{aligned}
& x\left(y_{d}, t\right) \approx \chi_{1}\left(y_{d}\right) \phi_{1}(t) \\
& \epsilon\left(y_{1}, t\right) \approx-\frac{h}{2} \times \frac{\partial^{2} \chi_{1}\left(y_{1}\right)}{\partial y^{2}} \phi_{1}(t)
\end{aligned}
$$

where $y_{1}$ is the location along the length of the beam in which the strain gage is attached. Since the motion is dominated by the first mode, the signal from one strain gage is sufficient for determining the displacement. In this case, the strain gage should be located away from $y=L$, such as near the camped end of the beam, for improved sensitivity because $\partial^{2} \chi(L) / \partial y^{2}=0$ [41].

\section{Controller Design}

Repetitive control is well-suited for tracking periodic trajectories [43], [44] as well as rejecting periodic disturbances [45], [46]. The control structure is based on the internal model principle. Compared to traditional proportional-integral (PI) or PID feedback controllers [4], where careful tuning is required and the residual tracking error persists from one operating cycle to the next, $\mathrm{RC}$ has the ability to reduce the error as the number of operating cycles increases [47]. It is pointed out that RC can also be "plugged into" to an existing feedback controller to enhance the tracking of periodic trajectories. The key feature of RC is

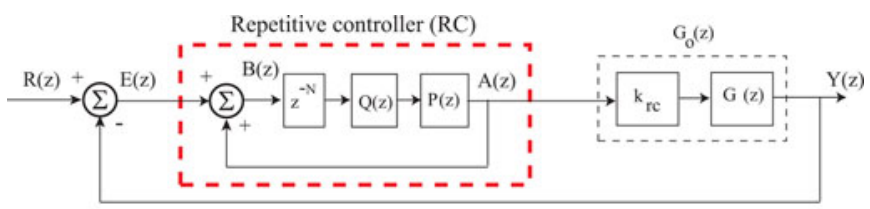

(a)

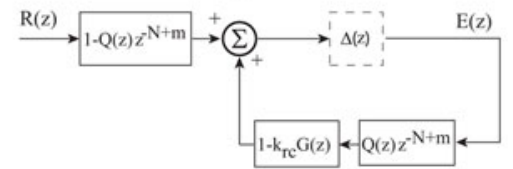

(b)

Fig. 11. (a) Block diagram of repetitive control system where the reference and output trajectories are $R(z)$ and $Y(z)$, respectively. The pure delay is denoted by $z^{-N}, Q(z)$ is a low-pass filter and $P(z)$ is a linear phase lead to enhance the performance. (b) Equivalent system.

a signal generator within the feedback loop (see Fig. 11) - the signal generator provides infinite gain at the fundamental frequency of the reference trajectory and its harmonics [43].

Let $R(z)$ be the $z$-transform of a given periodic reference trajectory with period $T_{p}$. The pure delay $z^{-N}$ within the inner positive feedback loop creates a signal generator, where $N=$ $T_{p} / T_{s}$ and $T_{s}$ is the sampling period. It is assumed that the inputoutput dynamics of the IPMC actuator is linear and represented by $G(z)$, where $z=e^{j \omega T_{s}}$, for $\omega \epsilon\left(0, \pi / T_{s}\right)$. The low-pass filter for stability and robustness is denoted by $Q(z)$, and $P(z)=z^{m}$ (where $m$ is a nonnegative integer) is a positive phase lead compensator to enhance the performance of the RC feedback system. Specifically, $P(z)$ provides $\theta(j \omega)=m T_{s}$ lead in phase.

By inspection, the transfer function of the signal generator that relates the signals $E(z)$ to $A(z)$ in Fig. 11 is given by

$$
\frac{A(z)}{E(z)}=\frac{Q(z) P(z) z^{-N}}{1-Q(z) P(z) z^{-N}}=\frac{Q(z) z^{(-N+m)}}{1-Q(z) z^{(-N+m)}}
$$

Without the low-pass filter $Q(z)$ and positive phase lead compensator $P(z)$, the poles of the signal generator are $1-z^{-N}=$ 0 . Therefore, the RC controller provides infinite gain at the harmonics of the periodic reference trajectory for tracking periodic trajectories. One drawback, however, is the large gain of RC at high frequencies that can lead to instability of the closed-loop system. Therefore, practical RC design incorporates a low-pass filter $Q(z)$ to address the stability and robustness issues. For simplicity, a low-pass filter of the form $Q(z)=a /(z+b)$, where $|a|+|b|=1$, is chosen. Alternatively, the zero-phase filter can also be considered [48].

Achieving a stable closed loop is one of the main challenges of RC. In the following, the conditions for stability are discussed. Let $H(z)=Q(z) z^{(-N+m)}$ and $G_{0}(z)=k_{r c} G(z)$, where $k_{\mathrm{rc}}$ is referred to as the $\mathrm{RC}$ gain. It is assumed that the reference trajectory $R(z)$ is periodic in time, and thus, has a discrete frequency spectrum. The transfer function relating the reference trajectory $R(z)$ and the tracking error $E(z)$ is

$$
S_{\mathrm{rc}}(z)=\frac{E(z)}{R(z)}=\frac{1-H(z)}{1-H(z)\left[1-G_{0}(z)\right]} .
$$




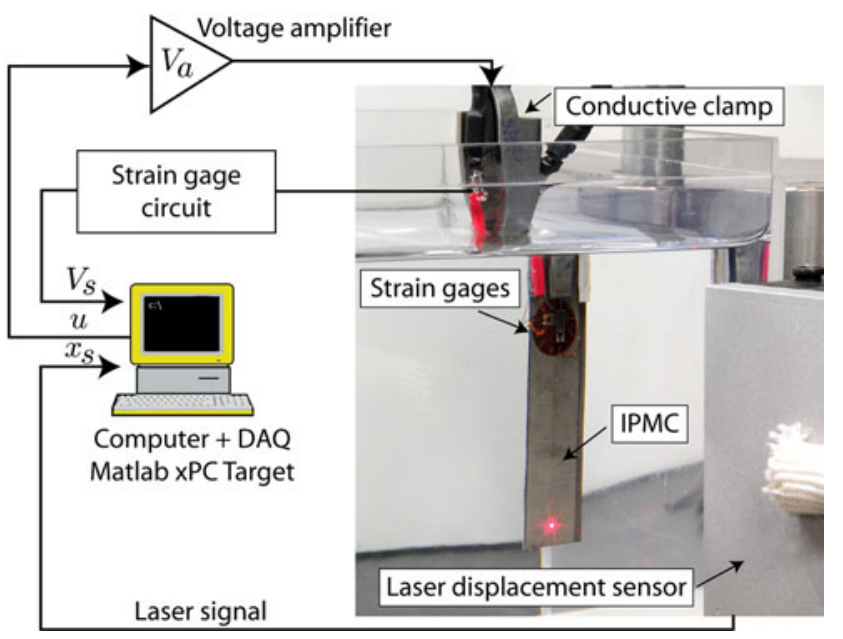

Fig. 12. Experimental setup for controlling the bending motion of the IPMC in water using PID and repetitive control.

By using (23), the RC block diagram in Fig. 11(a) is simplified to the equivalent interconnected system, as shown in Fig. 11(b). Replacing $z=e^{j \omega T_{s}}$, and since $1-H(z)$ is stable, the positive feedback system in Fig. 11(b) is asymptotically stable by the small gain theorem [49] when

$$
\left|H(z)\left[1-G_{0}(z)\right]\right|=\left|H\left(e^{j \omega T_{s}}\right)\left[1-k_{r c} G\left(e^{j \omega T_{s}}\right)\right]\right|<1
$$

for all $\omega \epsilon\left(0, \pi / T_{s}\right)$. Noting that $\left|Q\left(e^{j \omega T_{s}}\right)\right| \leq 1$

$$
\left|1-k_{r c} G\left(e^{j \omega T_{s}}\right)\right|<1 \leq \frac{1}{\left|Q\left(e^{j \omega T_{s}}\right)\right|}
$$

and setting the transfer function of the IPMC to $G\left(e^{j \omega T_{s}}\right)=$ $A(\omega) e^{j \theta(\omega)}$, where $A(\omega)>0$ and $\theta(\omega)$ are the magnitude and phase of $G\left(e^{j \omega T_{s}}\right)$, respectively, (24) is simplified to

$$
\left|1-k_{\mathrm{rc}} A(\omega) e^{j \theta(\omega)}\right|<1 .
$$

By observing that $e^{j \theta}=\cos (\theta)+j \sin (\theta)$ and requiring $k_{\mathrm{rc}}>$ 0 , (26) gives $1-2 k_{\mathrm{rc}} A(\omega) \cos [\theta(\omega)]+k_{\mathrm{rc}}^{2} A^{2}(\omega)<1$; hence

$$
0<k_{\mathrm{rc}}<\frac{2 \cos [\theta(\omega)]}{A(\omega)} \quad \text { and } \quad|\theta(\omega)|<\frac{\pi}{2} .
$$

Therefore, by picking a sufficiently small $k_{\mathrm{rc}}$, stability of the closed-loop system is guaranteed. It is noted that the lead compensator $P(z)$ compensates for the phase lag caused by the lowpass filter $Q(z)$, and thus, can be used to improve the tracking performance [50]. Because $N \gg m$, the modified delay $z^{-N+m}$ is causal and can easily be implemented digitally. Additionally, the RC gain $k_{\mathrm{rc}}$ can be adjusted to improve the closed-loop robustness and the rate of convergence of the tracking error.

\section{EXPERIMENTAL RESULTS AND DISCUSSION}

The experimental IPMC control system is shown in Fig. 12, where the actuator is submerged in deionized water. Two $0^{\circ} / 90^{\circ}$ tee-rosette strain gages are used, one bonded to the bottom and one bonded to the top surface of the actuator to form a half-bridge configuration. From the clamped end, the gages are located at $y_{1}=5 \mathrm{~mm}$. The effective length of the IPMC actuator

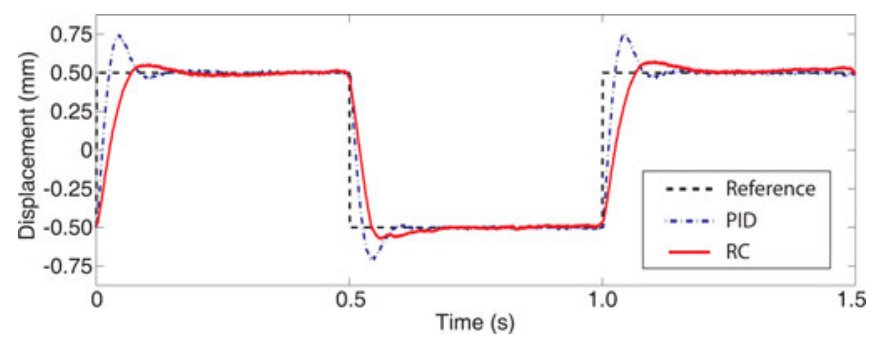

Fig. 13. Measuredsteady-state tracking results for square wave reference input at $1 \mathrm{~Hz}$.

is $L=45 \mathrm{~mm}$, and $w=15 \mathrm{~mm}$ and $h=0.5 \mathrm{~mm}$. The actuator's tip displacement is measured by the laser sensor pointing at approximately $y_{d}=42 \mathrm{~mm}$ from the fixed end. Based on the measured frequency response in Fig. 9, it is assumed that the bending motion is dominated by the first mode of vibration. It is also pointed out that the deformation range of the actuator is kept relatively small $( \pm 0.5 \mathrm{~mm})$ to avoid nonlinearities as well as nonlinearities associated with the laser displacement sensor that is used for comparison.

A linear dynamics model is obtained by curve-fitting the measured frequency response of the IPMC actuator, where the model is given by the following transfer function:

$$
G(s)=k_{0} \times \frac{\omega_{n}^{2}}{s^{2}+2 \zeta \omega_{n} s+\omega_{n}^{2}} \times\left(\frac{a}{s+a}\right)
$$

where $\zeta=0.067, \omega_{n}=62 \mathrm{rad} / \mathrm{s}, a=28$, and $k_{0}$ is the sensor gain. The model is shown by a dash-dot line in Fig. 9. A discrete-time model $G(z)$ is created by using MATLAB's " c2d" command with a sampling period of $T=1 \mathrm{~ms}$ for designing the two controllers in discrete time. It is noted that the dominant resonant frequency of the actuator (see Fig. 9) measured in water is approximately $9.87 \mathrm{~Hz}$.

The RC is first simulated in the MATLAB Simulink software, where the system is represented by the linear dynamics model $G(z)$ to determine the controller parameters prior to implementation. The RC parameters are chosen as $k_{\mathrm{rc}}=1.2$ and $m=6$ based on the discussion in Section IV. The pure delay is designed as $N=2000(0.5 \mathrm{~Hz}), N=1000(1 \mathrm{~Hz}), N=666$ $(1.5 \mathrm{~Hz})$, and $N=500(2 \mathrm{~Hz})$. For stability considerations, a low-pass filter $Q(z)$ with a cutoff frequency of $7 \mathrm{~Hz}$ is chosen. The controller is implemented by using MATLAB XPC Target environment using the sampling rate of $1 \mathrm{kHz}$. For comparison, a discrete-time PID controller is designed with gains $k_{p}=1.0$, $k_{i}=10$, and $k_{d}=0.01$, and closed-loop sampling frequency of $1 \mathrm{kHz}$. Both controllers use the strain gage signal for feedback information. In the first experiment, a square wave reference signal is applied to both controllers and the experimental results are shown in Fig. 13. The results are at steady state, recorded after the seventh period. The PID controller shows a response with significantly more overshoot, $50.0 \%$, compared to the RC with $14.9 \%$ overshoot. The settling time for both controllers is approximately the same at $200 \mathrm{~ms}$. It is noted that because the low-pass filter $Q(z)$ (used to ensure stability) has a cut-off frequency of $7 \mathrm{~Hz}$, there is a tradeoff between the performance of the RC and stability. 

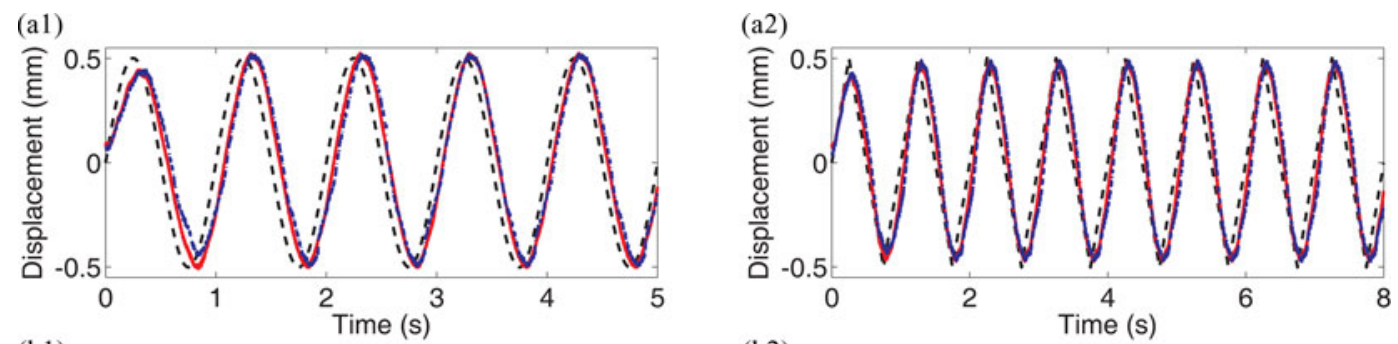

(b1)
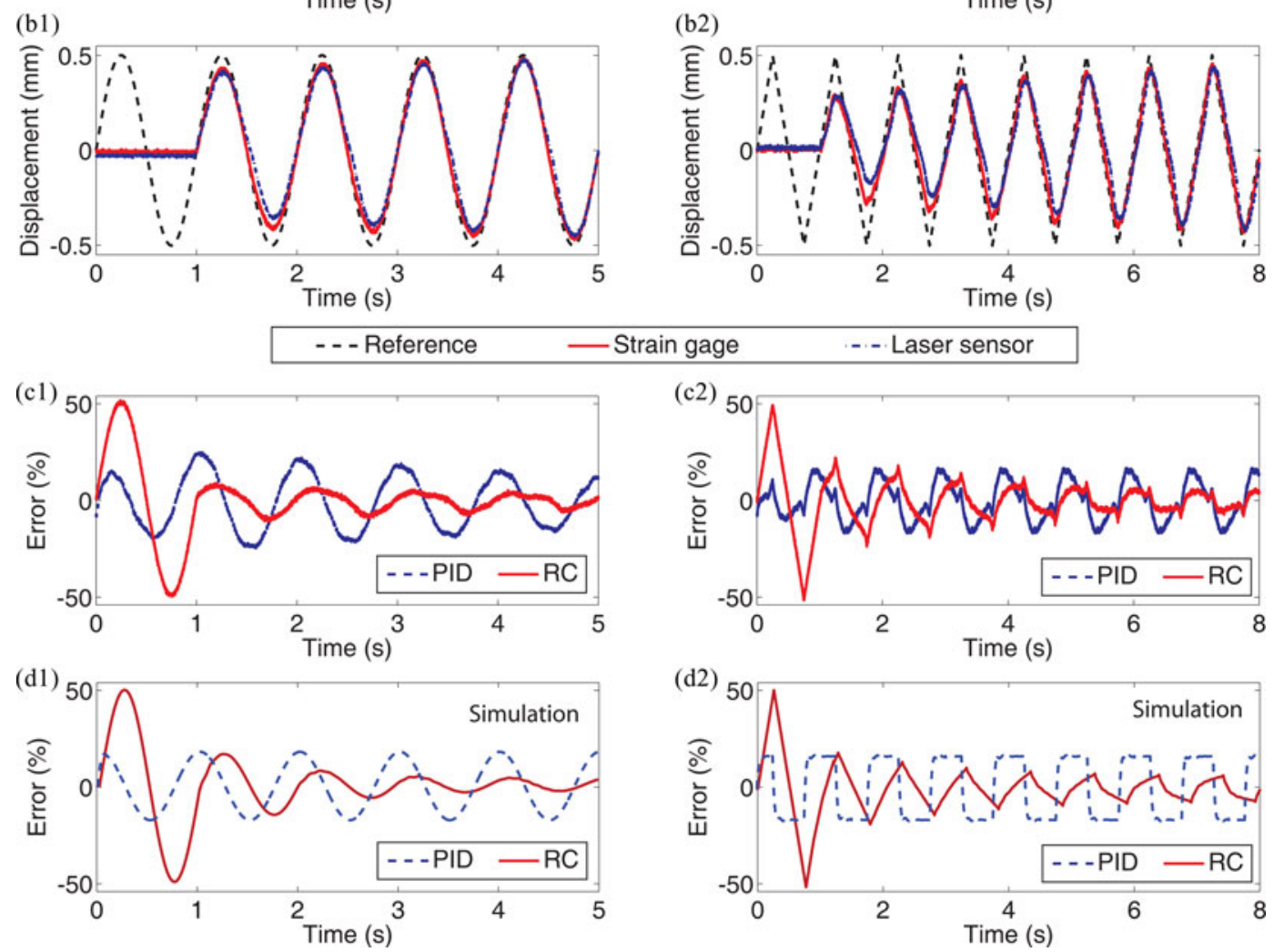

Fig. 14. Measured tracking results (1 Hz): sine wave tracking using (a1) PID and (b1) RC; triangle wave tracking using (a2) PID and (b2) RC; (c1) measured sine wave tracking error and (c2) triangle wave tracking error for PID and RC. Simulated tracking error for comparison: (d1) sine wave tracking error and (d2) triangle wave tracking error for PID and RC.

TABLE I

Comparison of TRacking Performance BetweEn PID AND RC FOR IPMC

\begin{tabular}{|c|c|c|c|c|c|c|c|c|c|}
\hline \multicolumn{2}{|c|}{} & \multicolumn{2}{c|}{$0.5 \mathrm{~Hz}$} & \multicolumn{2}{c|}{$1.0 \mathrm{~Hz}$} & \multicolumn{2}{c|}{$1.5 \mathrm{~Hz}$} & \multicolumn{2}{c|}{$2.0 \mathrm{~Hz}$} \\
\hline Cont. & Traj. & $e_{\max }(\boldsymbol{\%})$ & $e_{r m s}(\boldsymbol{\%})$ & $e_{\max }(\boldsymbol{\%})$ & $e_{r m s}(\boldsymbol{\%})$ & $e_{\max }(\boldsymbol{\%})$ & $e_{r m s}(\boldsymbol{\%})$ & $e_{\max }(\boldsymbol{\%})$ & $e_{r m s}(\boldsymbol{\%})$ \\
\hline PID & Sine & 9.6 & 5.8 & 16.0 & 9.8 & 17.2 & 11.6 & 23.0 & 15.9 \\
\hline PID & Triangle & 9.2 & 4.8 & 16.8 & 10.3 & 17.7 & 9.8 & 23.1 & 14.4 \\
\hline RC & Sine & 4.5 & 3.6 & 7.2 & 3.2 & 9.7 & 6.7 & 13.5 & 8.5 \\
\hline RC & Triangle & 4.9 & 2.2 & 7.3 & 3.6 & 9.7 & 4.1 & 13.3 & 8.5 \\
\hline
\end{tabular}

Next, sine and triangle reference signals with frequencies of $0.5,1.0,1.5$, and $2.0 \mathrm{~Hz}$ are investigated. In this case, the laser sensor signal is recorded and shown only for comparison. The measured tracking results (strain and laser sensor signals) and the tracking error at $1 \mathrm{~Hz}$ are shown in Fig. 14. Table I lists the maximum $\left(e_{\max }\right)$ and root-mean-squared $\left(e_{\mathrm{rms}}\right)$ tracking error for $0.5,1.0,1.5$, and $2.0 \mathrm{~Hz}$, computed as a percentage of the total output range $(1 \mathrm{~mm})$.

With the PID controller, the maximum tracking error for the sine and triangle reference trajectories are relatively large, for example, they exceed $16 \%$ at $1 \mathrm{~Hz}$ and above. At a scan rate of $2 \mathrm{~Hz}$, which is $20 \%$ of the first resonance, the tracking error for
PID control is $23 \%$. However, by applying the RC, the measured tracking error is reduced significantly, for example, at $0.5 \mathrm{~Hz}$, the maximum error with $\mathrm{RC}$ is $4.5 \%$ compared to the $9.5 \%$ with PID control. Therefore, RC reduces the tracking error by over $50 \%$, from $9.5 \%$ to $4.5 \%\left(e_{\mathrm{rms}}=3.6 \%\right)$. As the frequency of the reference signal increases beyond $2 \mathrm{~Hz}$, the behavior of the $\mathrm{RC}$ begins to deteriorate due to the fact that the controller gain reduces because of the $7 \mathrm{~Hz}$ cutoff frequency of the low-pass filter $Q(z)$. It is pointed out that because the $\mathrm{RC}$ is designed with a one-period delay (e.g., $N=1000$ for $1 \mathrm{~Hz}$ frequency), the controller begins to take effect after the first period, as shown in Fig. 14. Compared to the PID controller, the RC tracking 
error diminishes with each operating period, where steady-state behavior is achieved after approximately six cycles for tracking a triangle reference trajectory at $1 \mathrm{~Hz}$ (see Fig. 14). The response measured by the laser sensor closely matches the measurement from the strain signal, indicating the effectiveness of using the strain gage for displacement measurement and feedback control. The simulated response of the both controllers is compared in Fig. 14(d1) and (d2). For the sine wave reference input, the simulated tracking errors for the PID and RC closely match the measured tracking error in Fig. 14(c1). Likewise, for the triangle reference, the simulated tracking error for the RC agreed with the measured response. For the PID controller, the magnitude of the simulated error agrees with the measured response. It is pointed out that additional improvement in the tracking precision can be achieved by integrating the PID controller into the RC design, that is, replacing $k_{\mathrm{rc}}$ with a PID controller to further enhance the closed-loop performance. Likewise, an internal feedback loop can also be used to account for unmodeled dynamics such as the slow back-relaxation effect.

In summary, the experimental results demonstrate the effectiveness of using strain gages for sensing the motion of IPMC in water for feedback control. One unique advantage of resistive strain gages is compactness. The gages can be bonded to the surface of the IPMC or embedded into the polymer material. By incorporating additional gages arranged in appropriate orientations, both bending and twisting behaviors can be measured, and subsequently, used as feedback information. The measured response also shows that RC can be employed for tracking oscillatory reference trajectories in IPMCs for underwater applications, where the tracking error can be reduced by approximately $50 \%$ compared to PID control.

\section{CONCLUSION}

This paper discussed the application of resistive strain gages for displacement sensing and feedback control in IPMC actuators. Particularly, the displacement of the IPMC actuator was measured by a strain gage sensor bonded to the surface electrodes of the IPMC actuator. Strain gages are low cost, practical, effective, and importantly, compact compared to existing technologies for displacement sensing. The strain signal was used in the design of a discrete-time RC for tracking period reference trajectories in an IPMC actuator submerged in water. The tracking results showed that the combined control and sensing scheme can minimize the tracking error by approximately $50 \%$ compared to PID control for tracking of periodic signals including sinusoidal and triangular wave forms.

\section{ACKNOWLEDGMENT}

The authors like to thank S. Kim and Y. Jung of the Active Materials and Processing Laboratory for their help with fabricating the IPMC actuators used in the experiments; and M. Fleming of the Electroactive Systems and Controls Laboratory for his help with the adhesive experiments.

\section{REFERENCES}

[1] M. Shahinpoor and K. J. Kim, "Ionic polymer-metal composites: IV. Industrial and medical applications," Smart Mater. Struct., vol. 14, no. 1, pp. 197-214, 2005.

[2] E. Biddiss and T. Chau, "Electroactive polymeric sensors in hand prostheses: Bending response of an ionic polymer metal composite," Med. Eng. Phys., vol. 28, no. 6, pp. 568-578, 2006.

[3] W. J. Yoon, P. G. Reinhall, and E. J. Seibel, "Analysis of electro-active polymer bending: A component in a low cost ultrathin scanning endoscope," Sens. Actuators A, Phys., vol. 133, no. 2, pp. 506-517, 2007.

[4] B.-K. Fang, M.-S. Ju, and C.-C. K. Lin, "A new approach to develop ionic polymer-metal composites (IPMC) actuator: Fabrication and control for active catheter systems," Sens. Actuators A, Phys., vol. 137, no. 2, pp. 321-329, 2007.

[5] M. Aureli, V. Kopman, and M. Porfiri, "Free-locomotion of underwater vehicles actuated by ionic polymer metal composites," IEEE/ASME Trans. Mechatronics, vol. 15, no. 4, pp. 603-614, Aug. 2010.

[6] K. J. Kim, W. Yim, J. W. Paquette, and D. Kim, "Ionic polymer-metal composites for underwater operation," J. Intell. Mater. Syst. Struct., vol. 18, no. 2, pp. 123-131, 2007.

[7] N. Kamamichi, M. Yamakita, K. Asaka, and Z.-W. Luo, "A snake-like swimming robot using IPMC actuator/sensor," in Proc. IEEE Int. Conf. Robot. Autom., Orlando, FL, 2006, pp. 1812-1817.

[8] S. Guo, Y. Ge, L. Li, and S. Liu, "Underwater swimming micro robot using IPMC actuator," in Proc. IEEE Int. Conf. Mechatronics Autom., Luoyang, China, Jun. 25-28, 2006, pp. 249-254.

[9] H. Nakadoi, D. Sobey, M. Yamakita, and T. Mukai, "Liquid environmentadaptive IPMC fish-like robot using extremum seeking feedback," in Proc. IEEE/RSJ Int. Conf. Intell. Robot. Syst. (IROS), Nice, France, 2008, pp. 3089-3094.

[10] S. Guo, L. Shi, X. Ye, and L. Li, "A new jellyfish type of underwater microrobot," in Proc. IEEE Int. Conf. Mechatronics Autom., Harbin, China, 2007, pp. 509-514.

[11] Z. Chen, S. Shatara, and X. Tan, "Modeling of biomimetic robotic fish propelled by an ionic polymer-metal composite caudal fin," IEEE/ASME Trans. Mechatronics, vol. 15, no. 3, pp. 448-459, Jun. 2010.

[12] A. Menozzi, H. A. Leinhos, D. N. Beal, and P. R. Bandyopadhyay, "Openloop control of a multifin biorobotic rigid underwater vehicle," IEEE J. Ocean. Eng., vol. 33, no. 2, pp. 59-68, Apr. 2008.

[13] Z. Chen and X. Tan, "A control-oriented and physics-based model for ionic polymer-metal composite actuators," IEEE/ASME Trans. Mechatronics, vol. 13 , no. 5 , pp. 519-529, Oct. 2008.

[14] Z. Chen, D. Hedgepeth, and X. Tan, "A nonlinear control-oriented model for ionic polymer-metal composite actuators," Smart Mater. Struct., vol. 18, no. 5, pp. 1-9, 2009.

[15] Y. Fang, X. Tan, and G. Alici, "Robust adaptive control of conjugated polymer actuators," IEEE Trans. Control Syst. Technol., vol. 16, no. 4, pp. 600-612, Jul. 2008.

[16] S. Kang, J. Shin, S. J. Kim, H. J. Kim, and Y. H. Kim, "Robust control of ionic polymer-metal composites," Smart Mater. Struct., vol. 16, no. 6, pp. 2457-2463, 2007.

[17] Y. Shan and K. K. Leang, "Frequency-weighted feedforward control for dynamic compensation in ionic polymer-metal composite actuators," Smart Mater. Struct., vol. 18, no. 12, pp. 125016-1-125016-11, 2009.

[18] D. Pugal, K. Jung, A. Aabloo, and K. J. Kim, "Ionic polymermetal composite mechanoelectrical transduction: Review andperspectives," Polym. Int., vol. 59, no. 3, pp. 279-289, 2010.

[19] G. Alici, G. M. Spinks, J. D. Madden, Y. Wu, and G. G. Wallace, "Response characterization of electroactive polymers as mechanical sensors," IEEE/ASME Trans. Mechatronics, vol. 13, no. 2, pp. 187-196, Apr. 2008.

[20] K. Kruusamae, P. Brunetto, S. Graziani, A. Punning, G. Di Pasquale, and A. Aabloo, "Self-sensing ionic polymer-metal composite actuating device with patterned surface electrodes," Polym. Int., vol. 59, no. 3, pp. 300-304, 2009.

[21] A. Punning, M. Kruusmaa, and A. Aabloo, "A self-sensing ion conducting polymer metal composite (IPMC) actuator," Sens. Actuators A, Phys., vol. 136, pp. 656-664, 2007.

[22] Z. Chen, K. Ki-Yong, and X. Tan, "Integrated IPMC/PVDF sensory actuator and its validation in feedback control," Sens. Actuators A, Phys., vol. 144, no. 2, pp. 231-241, 2008.

[23] H.-H. Lin, B.-K. Fang, M.-S. Ju, and C.-C. K. Lin, "Control of ionic polymer-metal composites for active catheter systems via linear parameter-varying approach," J. Intell. Mater. Syst. Struct., vol. 20, no. 3, pp. 273-282, 2009. 
[24] B. C. Lavu, M. P. Schoen, and A. Mahajan, "Adaptive intelligent control of ionic polymermetal composites," Smart Mater. Struct., vol. 14, no. 4, pp. 466-474, 2005 .

[25] N. D. Bhat and W.-J. Kim, "Precision force and position control of ionic polymer-metal composite," J. Syst. Control Eng., vol. 218, no. 6, pp. 421432, 2004.

[26] C. Bonomo, L. Fortuna, P. Giannone, and S. Graziani, "A method to characterize the deformation of an ipmc sensing membrane," Sens. Actuators A, Phys., vol. 123/124, pp. 146-154, 2005.

[27] R. O. Riddle, Y. Jung, S.-M. Kim, S. Song, B. Stolpman, K. J. Kim, and K. K. Leang, "Sectored-electrode IPMC actuator for bending and twisting motion," presented at the SPIE Smart Structures and Materials Nondestructive Evaluation and Health Monitoring, San Diego, CA, 2010, vol. 7642 .

[28] Z. Chen and X. Tan, "Monolithic fabrication of ionic polymer-metal composite actuators capable of complex deformation," Sens. Actuators A, vol. 157 , pp. 246-257, 2010.

[29] X. Tan, D. Kim, N. Usher, D. Laboy, J. Jackson, A. Kapetanovic, J. Rapai, B. Sabadus, and X. Zhou, "An autonomous robotic fish for mobile sensing," in Proc. IEEE/RSJ Int. Conf. Intell. Robot. Syst., Beijing, China, 2006, pp. 5424-5429.

[30] K. J. Kim and M. Shahinpoor, "Ionic polymer-metal composites: II. Manufacturing techniques," Smart Mater. Struct., vol. 12, no. 1, pp. 65-79, 2003.

[31] P. Horowitz and W. Hill, The Art of Electronics, 2nd ed. New York: Cambridge Univ. Press, 1989.

[32] A. J. Fleming and K. K. Leang, "Integrated strain and force feedback for high performance control of piezoelectric actuators," Sens. Actuators A, Phys., vol. 161, no. 1/2, pp. 256-265, 2010.

[33] W. J. Manning, A. R. Plummer, and M. C. Levesley, "Vibration control of a flexible beam with integrated actuators and sensors," Smart Mater. Struct., vol. 9, no. 6, pp. 932-939, 2000.

[34] W. M. Murray and W. R. Miller, The Bonded Electrical Resistance Strain Gage. New York: Oxford Univ. Press, 1992.

[35] C. T. Wu, "Transverse sensitivity of bonded strain gages," Exp. Mech., vol. 2, no. 11, pp. 338-344, 1962.

[36] A. Chernov, "Determination of transverse sensitivity of resistor strain gages," Meas. Tech., vol. 34, no. 3, pp. 249-253, 1991.

[37] S. Nemat-Nasser, "Micromechanics of actuation of ionic polymer-metal composites," J. Appl. Phys., vol. 92, no. 5, pp. 2899-2915, 2002.

[38] W. A. Lughmani, J. Y. Jho, J. Y. Lee, and K. Rhee, "Modeling of bending behavior of IPMC beams using concentrated ion boundary layer," Int. J. Precis. Eng. Manuf., vol. 10, no. 5, pp. 131-139, 2009.

[39] M. E. Tuttle and H. Brinson, "Resistance-foil strain-gage technology as applied to composite materials," Exp. Mech., vol. 24, no. 1, pp. 54-65, 1984.

[40] A. C. Pisoni, C. Santolini, D. E. Hauf, and S. Dubowsky, "Displacements in a vibrating body by strain gauge measurements," in Proc. 13th Int. Conf. Modal Anal., 1995, pp. 119-125.

[41] C.-J. Li and A. G. Ulsoy, "High-precision measurement of tool-tip displacement using strain gauges in precision flexible line boring," Mech. Syst. Signal Process., vol. 13, no. 4, pp. 531-546, 1999.

[42] D. Inman, Engineering Vibration, 2nd ed. Upper Saddle River, NJ: Prentice-Hall, 2001

[43] T. Inoue, M. Nakano, and S. Iwai, "High accuracy control of a proton synchrotron magnet power supply," in Proc. 8th IFAC World Congr., 1981, vol. 20, pp. 216-221.

[44] S. Hara, Y. Yamamoto, T. Omata, and M. Nakano, "Repetitive control system: A new type servo system for periodic exogenous signals," IEEE Trans. Autom. Control, vol. 33, no. 7, pp. 659-668, Jul. 1988.

[45] E. J. Solcz and R. W. Longman, "Disturbance rejection in repetitive controllers," Adv. Astronaut. Sci., vol. 76, no. 3, pp. 2111-2130, 1992.

[46] G.-L. Chen and G. T.-C. Chiu, "Spatially periodic disturbance rejection with spatially sampled robust repetitive control," Trans. ASME, J. Dyn. Syst. Meas. Control, vol. 130, no. 2, pp. 021002-1-021002-11, 2008.

[47] K. K. Chew and M. Tomizuka, "Digital control of repetitive errors in disk drive systems," IEEE Control Syst. Mag., vol. 10, no. 1, pp. 16-20, Jan. 1990

[48] M. Tomizuka, "Zero-phase tracking algorithm for digital control," Trans. ASME, J. Dyn. Syst. Meas. Control, vol. 109, pp. 65-68, 1987.

[49] K. Zhou and J. C. Doyle, Essentials of Robust Control. Englewood Cliffs, NJ: Prentice-Hall, 1998.

[50] H. L. Broberg and R. G. Molyet, "A new approach to phase cancellation in repetitive control," in Proc. IEEE Ind. Appl. Soc. Annu. Meeting, Oct. 1994, vol. 3, pp. 1766-1770.

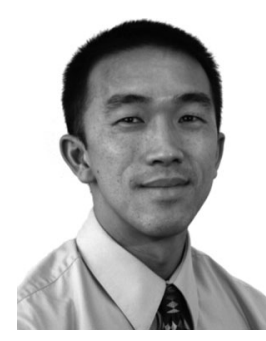

Kam K. Leang received the B.S. and M.S. degrees in mechanical engineering from the University of Utah, Salt Lake City, in 1997 and 1999, respectively, and the $\mathrm{Ph} . \mathrm{D}$. degree from the University of Washington, Seattle, in 2004.

$\mathrm{He}$ is currently an Assistant Professor in the Mechanical Engineering Department, University of Nevada, Reno, which he joined in 2008. From 2005 to 2008, he was with the Mechanical Engineering Department, Virginia Commonwealth University, Richmond. His current research interests include modeling and control of piezoactuators for scanning probe microscopy applications, fabrication and control of electroactive polymers, and design of MEMS for nanotechnology.

Dr. Leang is a member of the American Society of Mechanical Engineers (ASME) and the International Society for Optical Engineers (SPIE).

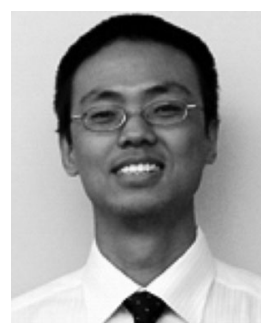

Yingfeng Shan received the M.S. degree in mechanical engineering from Virginia Commonwealth University, Richmond, in 2008. He is currently working toward the Ph.D. degree at the University of Nevada, Reno.

From 2005 to 2006, he was a Control Engineer at China National Petroleum Corporation, China. His current research interests include modeling and precision control of active material systems such as piezoactuators and ionic polymer metal composite actuators.

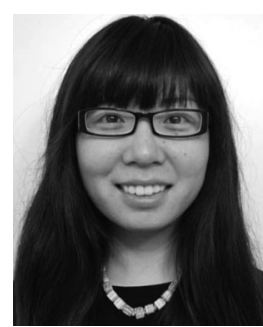

Sisi Song received the B.S. degree in mathematics in 2009 from the University of Nevada, Reno, where she is currently working toward the M.S. degree in mathematics.

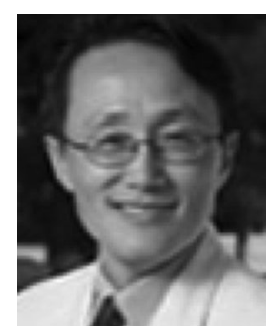

Kwang J. (Jin) Kim received the Graduate degree from Yonsei University, Seoul, Korea, in 1987, and the M.S. and Ph.D. degrees from Arizona State University (ASU), Phoenix, in 1989 and 1992, respectively.

He is currently a Professor and the Chair of the Mechanical Engineering Department and the Director of the Active Materials and Processing Laboratory (AMPL) and the Low Carbon Green Technology Laboratory (LCGTL), University of Nevada, Reno. From 1993 to 1995 , he was a Postdoctoral researcher at the University of Maryland, College Park. He has authored or coauthored more than 270 technical papers and two books and holds three patents. His current research interests include a broad spectrum of active materials/sensors and (renewable) energy systems.

(ASME) 